Morad Montazami*

\title{
Ornements transfrontières/avant-gardes marocaines : entre ésotérisme et post-colonialisme
}

\author{
Ornaments without boundaries/Moroccan avant-gardes: \\ between esotericism and post-colonialism
}

DOI 10.1515/asia-2016-0048

\begin{abstract}
The discourses surrounding the ornament in Western modernist and formalist art theory (Riegl, Focillon) encourage such ideas as the emancipation of the ornament. The same preoccupation has been followed - from a totally different perspective - by Moroccan avant-garde painters (Ahmed Cherkaoui, Farid Belkahia and the Casablanca School) who stood up for the decolonization of arts and culture. How can we retrace the visual and literary genealogy (in practice and theory) that led to this esoteric use of the ornament as a tool for mobility and migration through strategies of sign making and identity politics?
\end{abstract}

Keywords: Ornament, Moroccan avant-garde, body, Casablanca School, colonial memory

\section{Ornement émancipé}

On sait la répugnance d'une certaine doxa moderniste européocentrée vis-à-vis de l'ornement et du motif dit " décoratif ». Nul emblème plus vivace à ce rejet que le grand opposant à la Sécession viennoise et à l'Art Nouveau, l'architecte et théoricien Adolf Loos, dans son essai Ornement et crime écrit en 1908. ${ }^{1}$ Celui qui se félicitait d'avoir « libéré l'humanité de l'ornement superflu » n'avait sûrement pas prévu de devenir un repoussoir contre lequel mille et une vies nouvelles de

1 Sur Adolf Loos voir « L'ornement comme crime ou comme style » dans Buci-Glucksmann 2008.

*Corresponding author: Morad Montazami, Tate Modern for the Middle East and North Africa supported by Iran Heritage Foundation - TATE MODERN, Bankside, London SE1 9TG, UK.

E-mail: montazami@hotmail.com 
l'ornement étaient prêtes à éclore. Or il est d'autant plus remarquable qu'à celles de Vienne, Paris et Bruxelles se soient ajoutées celles moins connues de Rabat, Casablanca et Marrakech, comme je voudrais l'exposer dans cet article à travers les artistes Ahmed Cherkaoui ${ }^{2}$ (1934-1967) et Farid Belkahia ${ }^{3}$ (1934-2014) en priorité. Entendu que la contre-attaque de l'ornement s'est étendue quasiment dans tout le Moyen-Orient et l'Afrique, d'Alger à Beyrouth en passant par Téhéran et le Caire, en recourant à différents vocables et labels, tels que Saqqakhaneh, Hurûfiyya ou encore " peinture du signe ", " école du signe arabe "; c'est-à-dire aussi bien des mouvements artistiques nationaux et localisés que des tendances esthétiques transnationales, panarabes ou encore tiers-mondistes ; et ce, des années 1950 aux années 1980. ${ }^{4}$

Dès lors le choix de s'arrêter sur l'expérience marocaine comme paradigme se justifie en vertu, justement, de son caractère intrinsèquement transculturel (Maroc berbère, Maroc arabe, Maroc islamique, Maroc africain, Maroc méditerranéen...) et de sa synthèse particulièrement efficace entre " tradition » et «modernité " mais aussi, fait remarquable, entre pratique et théorie. Si l'ornement est accessoire, parure, ajout, excès il peut aussi bien être symbole, symptôme, moteur, algorithme. Un enjeu théorique majeur s'annonce en réalité sous les auspices de ce dilemme entre signe de luxe et signe de complexité, comme le revendique pertinemment l'historien de l'art Thomas Golsenne : « Comment penser l'œuvre ornementale de façon positive, le remplissage non comme un excès ni un processus naturel, mais comme une puissance expressive et, par là, signifiante ${ }^{5}$ ?"

2 Né en 1934 à Boujad, Ahmed Cherkaoui, diplômé de l'Ecole des arts et métiers de Paris en 1959, s'inscrit ensuite à l'Ecole nationale supérieure des Beaux-arts dans l'atelier d'Aujame qu'il quitte en 19 61.Il passe ensuite un an à l'Académie de Varsovie en Pologne. De retour à Paris, il obtient une bourse de l'UNESCO qui lui permet d'effectuer des recherches sur le signe berbère et la calligraphie arabe. Il est considéré avec Jilali Gharbaoui comme un des fondateurs de la peinture abstraite au Maroc.

3 Né en 1934 à Marrakech, Farid Belkahia fait ses études à l'Ecole des Beaux-Arts à Paris en 1959 et à l'Académie de Théâtre à Prague. Directeur de l'Ecole des Beaux-Arts de Casablanca, entre 1962 et 1974, il rénove l'enseignement en s'entourant d'une équipe de pionniers (Melehi, Chabâa, Azema, Hamidi, Hafid, Maraini, Flint). Après sa participation à la revue Souffles, il développe à la fois la notion d'art pour l'espace public et son langage individuel qui prend forme dans ses œuvres en cuivre, sur peau et autres matières " vivantes ».

4 Nous renvoyons sur ces questions et appellations aux recherches pionnières de Silvia Naef, notamment sur le courant esthétique hurufiyyâ, dans Naef 1996 ainsi que Shabout 2007.

5 Voir Golsenne 2010: 112-113. 

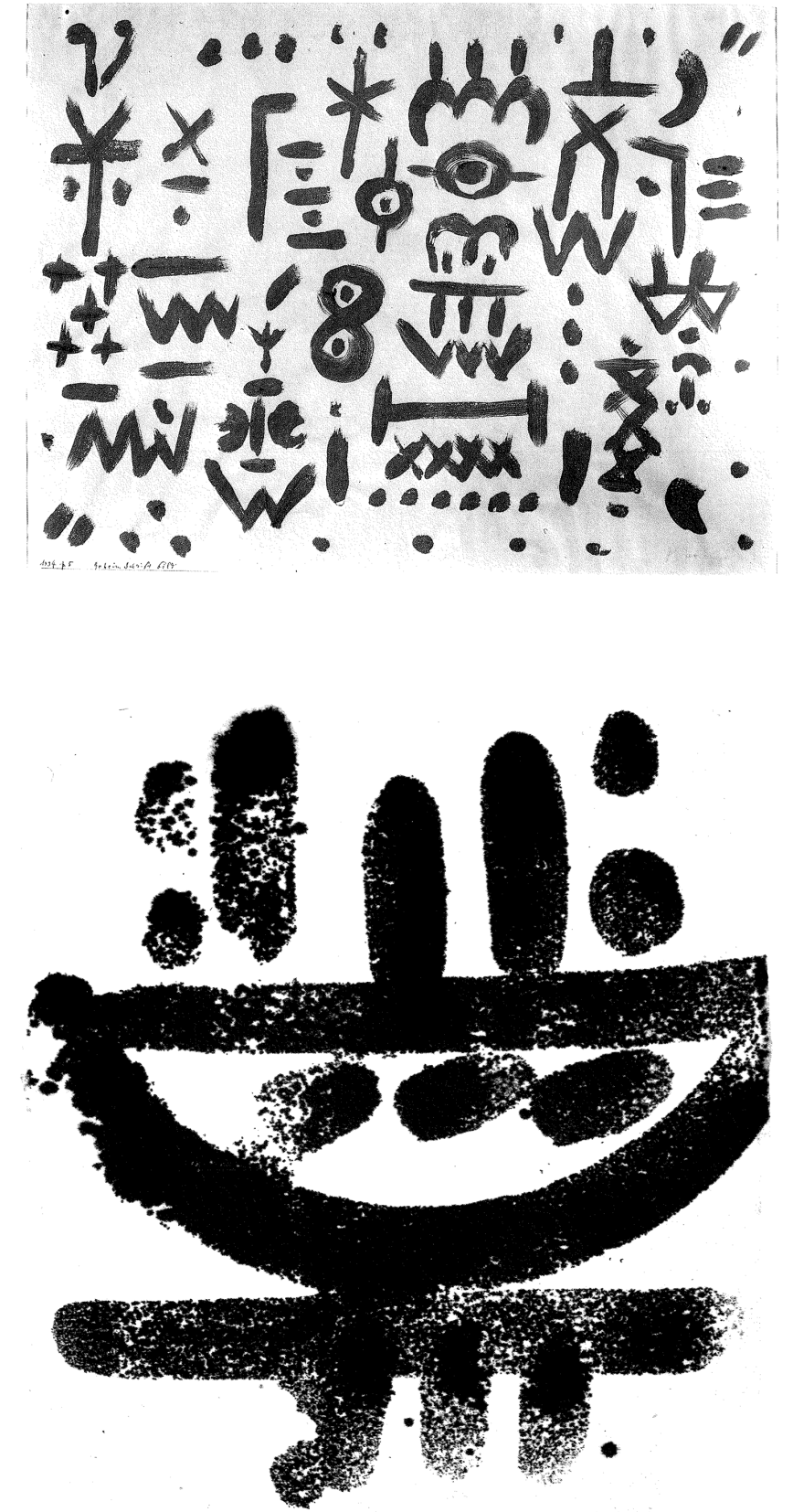

Figure 1: Paul Klee, Geheimschriftbild (Cryptogramme), 1934. Peinture à la colle sur papier et carton, $48 \times 63,5 \mathrm{~cm}$. Fondation Paul Klee, Berne.

Figure 2: Ahmed Cherkaoui, Sans titre, 1965. Dessin à l'encre de chine sur papier, $13 \times 16 \mathrm{~cm}$. Collection Noureddine Cherkaoui. Photo: P. Maillard. 

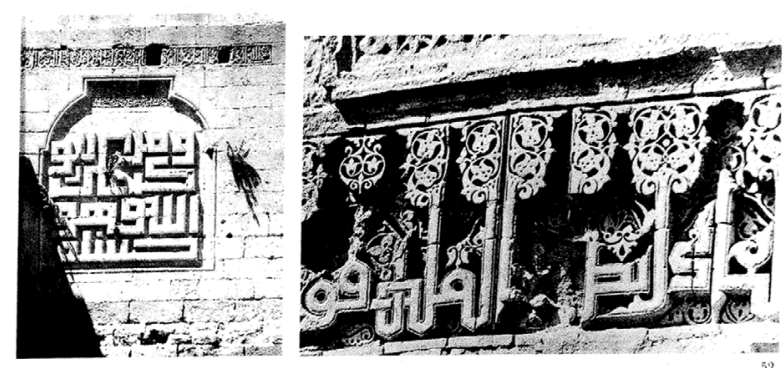

51 51. Turquie, Mardin, inscription sur pierre dans la mosquée, XII siècle.

52. Iran, Khargird, stuc de la madrasa de Nizamiyah, fin du XI' siècle.

53. Iran, mosquéc de Linjan (près d'Ispahan), XII siècle : panneau portant le nom des descendants du Prophète, stuc gravé ou moulé, vers 1300 .

54. Lettres animées en argent incrusté sur bronze.
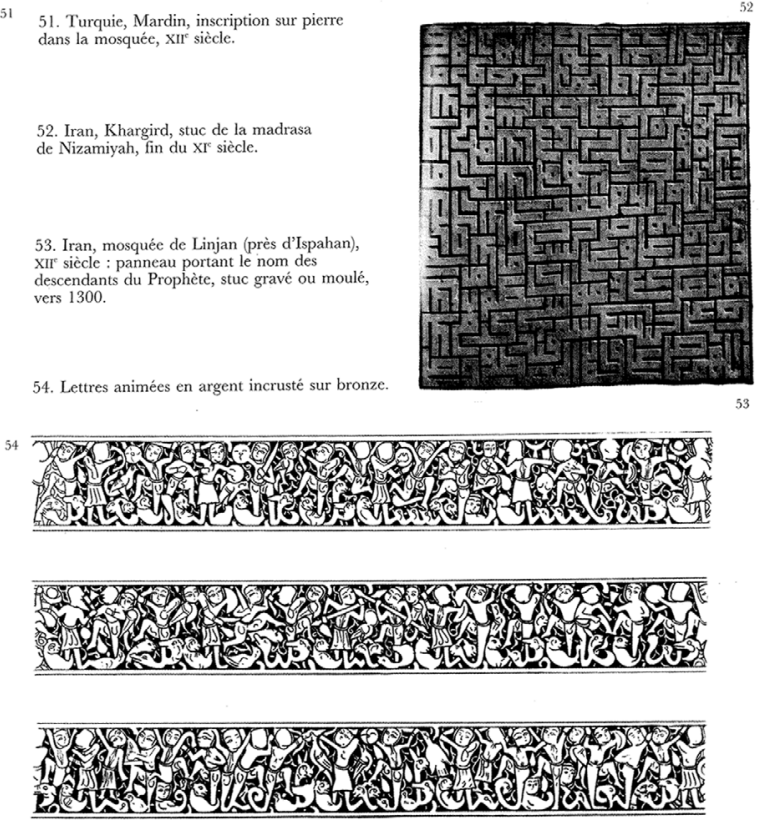

Figure 3: Illustrations publiées dans Oleg Grabar, L'ornement. Formes et fonctions dans l'art islamique, Paris, Flammarion, 1996 (1992). Exemples d'écritures gravées sur pierre ou stuc en Turquie et en Iran (11-12 $2^{\mathrm{e}}$ siècle). Exemple de frise ornementale en lettres animées et anthropomorphes, en argent incrusté sur bronze.

On revisitera à cet effet une généalogie d'auteurs " ornementalistes " plus anciens, historiens de l'art, architectes ou philosophes. Souvent qualifiés de " formalistes », ils balisèrent ce terrain de leurs études, encourageant à une certaine émancipation de l'ornement (Owen Jones, Alois Riegl, Henri Focillon...). Une émancipation qui devait se produire au travers des mailles habituellement tissées entre arts majeurs et arts " mineurs ", ou entre une vision de l'histoire guidée par les noms prestigieux des grands créateurs contre une vision de 


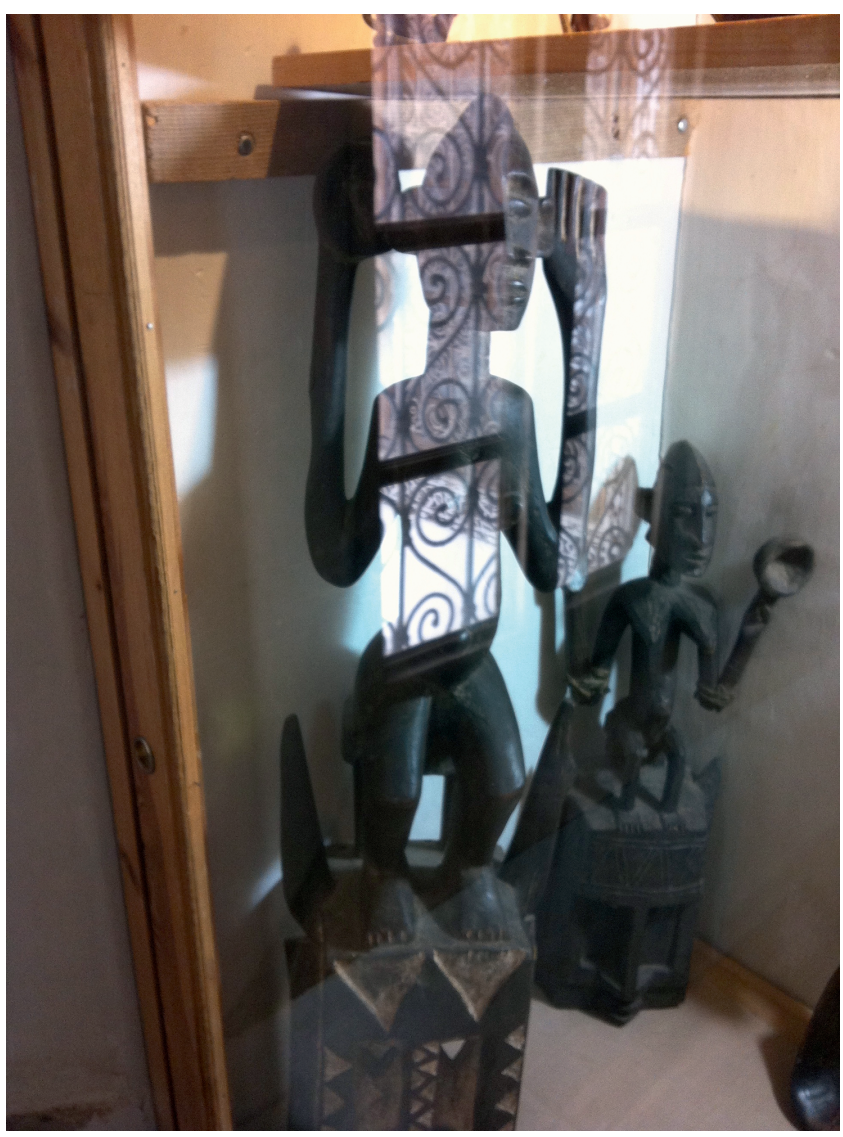

Figure 4: Vitrine des statues africaines (Mali) au musée Bernt Flint ou musée Tiksiwin, Marrakech, 2014. Photo de l'auteur. Les ornements des fenêtres se reflètent sur les statues superposant époques et influences diverses.

l'histoire née dans les langages techniques les plus anonymes (tapis, couture, céramique, vitrail...). Ces formes à la fois savantes et populaires mais excédant le cadre admis des « Beaux-arts » qui perdure assez largement jusqu'à la période de décolonisation.

Ce retour de l'ornement s'origine à la fois dans une psychologie de l'art universaliste et dans un certain " orientalisme » (celui de l'académisme plutôt que du colonialisme). Cette généalogie passe par Aloïs Riegl qui, pour comprendre l'évolution de l'ornementation végétale, nous fait traverser en un seul voyage les civilisations égyptienne, mésopotamienne, phénicienne et perse avec l'ornement pour pierre philosophale. Ce dernier devient un opérateur 


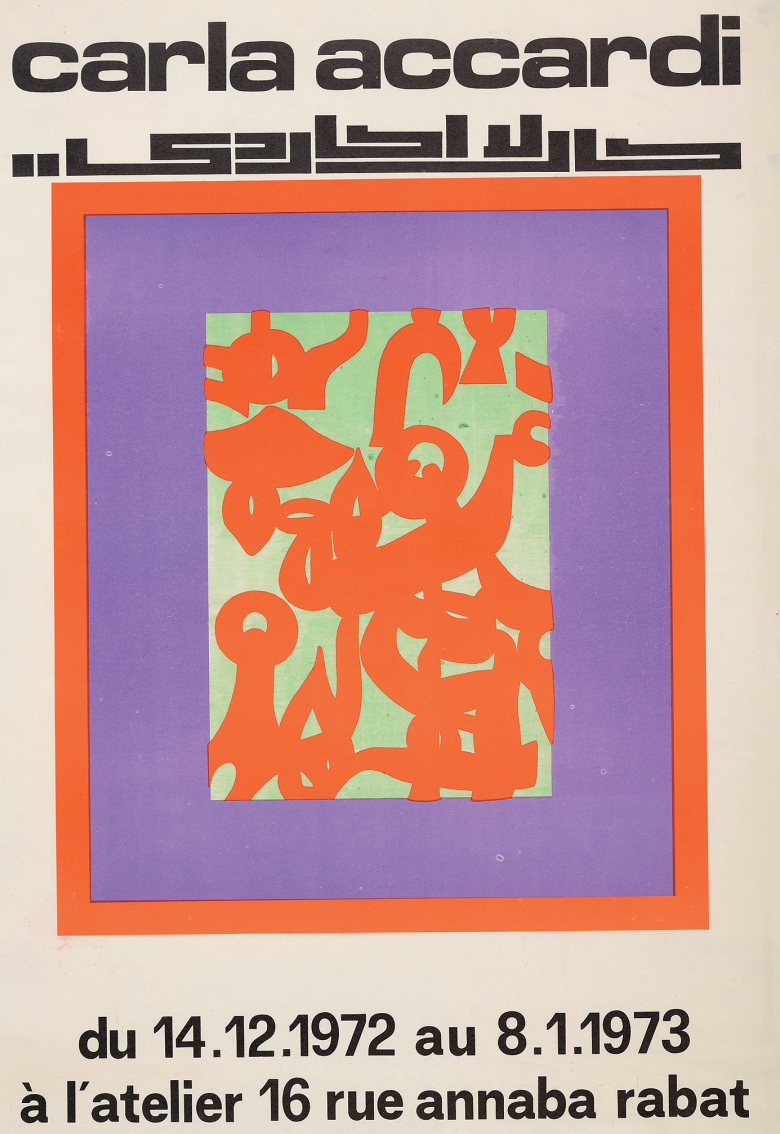

Figure 5: Affiche de l'exposition Carla Accardi, galerie L'Atelier, 1972-1973. Archives Pauline de Mazières.

intrinsèque de migration et de translitération des formes. On en passera aussitôt, pour délier un peu plus le fil du discours sur l'ornement, par l'ouvrage non moins fameux d'Henri Focillon, Vie des formes, de 1934. On peut y lire cette définition, où, à peu de chose près, Focillon parle de l'ornement comme il parlerait d'une carte ou d'un paysage ; plutôt en termes de dynamiques que de simples fonctions :

Alphabet de la pensée humaine aux prises avec l'espace [...] selon qu'il est pierre, bois, bronze ou trait de pinceau [...] un lieu de spéculation très étendue et comme un observatoire [...] de la vie des formes dans leur espace [...] la flexion d'une courbe, un rinceau, qui 


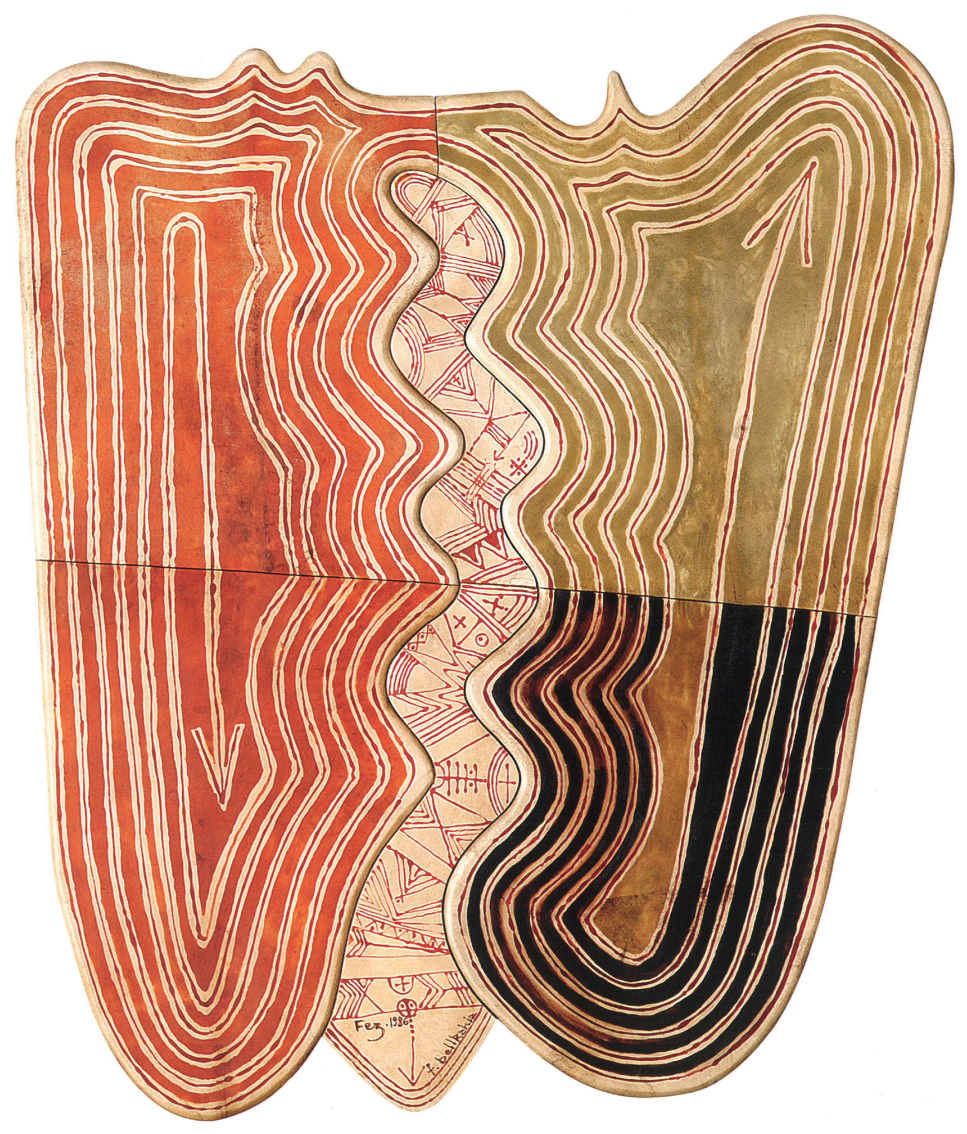

Figure 6: Farid Belkahia, Labyrinthe, 1986. Tenture sur peau, $140 \times 134 \mathrm{~cm}$. Fondation Belkahia, Marrakech.

implique tout un avenir de symétries, d'alternances, de dédoublements, de replis, chiffre déjà le vide où il paraît et lui confère une existence inédite. Réduit à un mince trait sinueux, [l'ornement] est déjà une frontière et un chemin. ${ }^{6}$

L'enjeu va en fait plus loin que l'incroyable pouvoir de translittération et de prolifération de l'ornement, à commencer par le domaine architectural où il s'exprime avec le plus de fluidité (comme des motifs d'entrelacs végétaux et des structures géométriques sur une mosquée d'Ispahan ou un palais de Sana que l'on retrouve presque à l'identique sur un palais de Venise ou une église de

6 Focillon 2004: 27. 
Palerme). Comment ces phénomènes saisissants de comparatisme transculturel et de " migration » du motif, hérités notamment de Riegl et de Focillon, ${ }^{7}$ vont-ils se trouver rejoués par des artistes comme Cherkaoui ou Belkahia, dans un tout autre contexte, dont il sera question ici ? Comment ces derniers ont-ils su renouer avec la subjectivité de l'ornement, pour mettre au travail sa puissance expressive et signifiante ? Une subjectivité allant bien au-delà de celui ou celle qui a conceptualisé ou dessiné tel ornement. Enfin, comment les avant-gardes marocaines des années 1960 ont-elles pu constituer le chaînon manquant et nécessaire pour déjouer la conception occidentale de l'ornement ? Largement illustrée par le colonialisme mais remontant aux philosophes de l'Antiquité, celle-ci procède d'abord d'une pensée platonicienne, essentialiste et anthropocentrique. Son mythe fondateur est celui de l'horror vacui (ou horreur du vide) dans lequel l'ornement ne fait que « remplir » le vide ; non seulement synonyme de débauche, d'exubérance, mais aussi de primitivité et de manque de rationalité. Une conception, en définitive, qui milite contre la menace idéologique $d u$ " kitsch $^{8}$ » risquant d'altérer à la fois le système des Beauxarts et celui du modernisme des avant-gardes historiques. Un combat perdu d'avance, pourrait-on dire, rétrospectivement.

On trouvera ainsi matière à interroger quelque dialogue insoupçonné entre le modernisme européen et la génération postindépendance des artistes notamment marocains, à travers l'ornement. Les grands noms que sont Matisse ou Paul Klee, forts de leurs voyages au Maroc ou en Tunisie, y puisent certains ornements pour les intégrer à leur propre système de représentation. Pour ce faire, ils n’ont nul besoin de procéder à un examen approfondi de la chaîne signifiante, du répertoire symbolique ou de l'environnement desquels ces ornements sont prélevés. Mais le territoire de l'œuvre d'art est lui bien remis en question par la réappropriation postcoloniale de l'ornement, comme nous le verrons. Alors que pour Matisse ou Klee l'ornement reste principalement un principe d'organisation de l'espace pictural, pour Farid Belkahia ou Ahmed

7 Il n'est pas indifférent que Riegl comme Focillon aient occupé des fonctions au sein d'institutions muséales. Riegl comme conservateur du musée des arts appliqués de Vienne entre 1886 et 1897 et Focillon comme directeur du musée des Beaux-arts de Lyon entre 1913 et 1924. Ce qui dénote un rapport intime à l'objet, sa conservation comme sa mise en scène mais aussi une pragmatique de l'espace.

8 Pour une brève histoire des idées entourant la notion européocentrée d'horror vacui voir Golsenne 2012. A propos de la notion de kitsch, Golsenne rappelle par ailleurs que dans la deuxième moitié du $19^{\mathrm{e}}$ siècle "l'apparition du kitsch ne signifie pas encore le mauvais goût, mais une esthétique intermédiaire entre l'artisanat à l'ancienne et la production industrielle, tradition et modernité ». 
Cherkaoui l'ornement est un principe de vie, guidant une certaine morale du geste pictural plutôt que la simple composition qui en ressort.

On peut estimer que les tournants postindépendance et plus généralement postcolonial impliquent pour nombre d'artistes issus de pays arabes (qu'ils se soient formés en Europe ou pas) de surmonter un double dispositif, l'un pédagogique et l'autre esthétique : d'une part, celui de l'académisme et son « école des Beaux-arts » importés de France ou d'Espagne (au Caire dès 1908, à Tétouan en 1945), se diffusant également par les salons de peinture et autres cercles de " connaisseurs ", colonisateurs en majorité, renvoyant volontiers les artistes locaux au domaine du folklore et de la peinture dite " naïve ». ${ }^{9}$ D'autre part, celui d'un orientalisme tardif (qui commence avec Delacroix et se termine avec Matisse) ; un orientalisme à fortiori issu des avant-gardes et non des élites ; qui ne relève pas de l'« Orient "stricto sensu et qui peut se retrouver dans la fascination de Josef Albers pour la civilisation aztèque ; un orientalisme à la fois formaliste et introspectif, qui relève souvent de l'autoanalyse (notamment dans le cas de Paul Gauguin ou de Paul Klee pour des raisons très différentes) ; une manière de collectionner les signes et de traduire la " palette » chromatique du pays, ces signes réfugiés au coin d'un geste, un fragment de mythe, un rayon de soleil à travers une fenêtre, une parcelle d'architecture (on pense, en littérature, au Roland Barthes de L'Empire des signes) ; un orientalisme paradoxal qui en définitive ne sert plus à mettre en scène ou objectiver " l'Arabe », « l'islam » et autres « fantasmes d'Orient » mais à explorer subjectivement la fluidité d'une certaines frontière physique, psychologique et culturelle. ${ }^{10}$

L'ornement est comme la boussole au centre de ces échanges conscients et inconscients, que ce soit par relégation sociale dans le dispositif académique ou par revalorisation philosophique dans le dispositif moderniste.

\section{Modernisme rédempteur}

Que ce soit au Caire dès la fin des années 1930 avec le groupe " Art et liberté » des dits « surréalistes » égyptiens (George Henein, Ramsès Younan, Kamel el-

9 Mentionnons cependant que dans le cas de l'Egypte, les élites locales, à l'origine des salons et des cercles artistiques, jouent un rôle tout aussi significatif que l'influence étrangère.

10 Cet orientalisme paradoxal, dans le champ visuel, fait fortement écho au phénomène d'hybridité étudié par Abdelkébir Khatibi dans le champ littéraire. Rassemblant Aragon, Duras, Barthes, Ollier, Genet et Segalen, il perçoit chez ces auteurs un détournement des clichés exotiques habituels par le biais d'un rapport initiatique au voyage, confinant à l'autoanalyse. Voir Khatibi 1987. 
Telmissany), le Groupe de Bagdad pour l'art moderne dans les années 1950 sous l'impulsion de Jawad Salim, ou encore dans les années 1960 avec les artistes de l'Ecole de Casablanca, on peut dire que les avant-gardes anticoloniales et postcoloniales s'activent sur un terrain multiple, panarabe, panafricain et transméditerranéen. L'objectif commun découle d'un renversement de valeurs largement inspiré des idées marxistes, internationalistes et anticoloniales. L'Ecole de Casablanca connaît sa mue postcoloniale avec des artistes tels que Mohamed Melehi et Mohamed Chabâa, sous la direction de Farid Belkahia, c'està-dire entre 1962 et 1974. Il est remarquable dans ce cas de voir comment " école » au sens institutionnel et " école » au sens de l'avant-garde ou du « groupe d'artistes » se sont télescopés, prenant là sans doute son originalité première.

« L'Ecole de Casa », vers laquelle les historiens de l'art se tournent de plus en plus, s'érige en exemple du retour à Soi ou du retour à la tradition comme expérimentation avec la subjectivité moderne.

L'organe fédérateur est alors une revue intitulée Souffles, organisée entre autres par le poète Abdelatif Laabi, qui parut entre 1966 et 1973, portée par toute une génération d'artistes, poètes, peintres et cinéastes désireux de refonder une politique culturelle nationale. ${ }^{11}$ Aventure qui commence exactement à la mort d'un artiste peintre fondamental, Ahmed Cherkaoui. Ce dernier, avec son décès prématuré, acquiert le statut de précurseur posthume dans la recherche sur l'interstice et l'extension du domaine de la lettre, eu égard de son ancrage dans la tradition islamique, soufie ou encore berbère et versé dans la pratique de la calligraphie, bien qu'il s'en émancipera totalement. Son engagement pourrait se résumer ainsi au travers des objectifs affichés par Abdellatif Laâbi dans le premier numéro de Souffles:

Pour Souffles, la culture populaire est le ciment de la nation, car elle est accessible à tous et est porteuse de ce qui fait l'originalité intrinsèque du pays. D'où le potentiel de modernité, voire le caractère révolutionnaire des tapis, des bijoux, du patrimoine oral, des danses, des contes, ou encore de l'architecture. Il s'agissait donc d'en restaurer la vitalité, de débarrasser cette culture de ses éléments archaïques pour lui permettre de déployer tout son potentiel créateur, jugé seul à même de faire accéder la création marocaine à l'universalité.

Plus qu'un simple retour à la tradition, l'expérience qui se dessine, dès le début des années 1960, pour les esprits anticonformistes au Maroc, ouvre surtout sur

11 Un ouvrage fait désormais référence sur le sujet, celui de Kenza Sefrioui (2013): La revue Souffles. 1966-1973. Espoirs de révolution culturelle au Maroc. Casablanca: Sirocco. Cf. également l'article de Marion von Osten dans ce même dossier. 
une subjectivation des arts populaires et de l'ornement - qui devient lieu de passage entre les tapis, les bijoux, la danse, l'architecture. Une attitude parfaitement incarnée par Ahmed Cherkaoui qui se propose de prendre le tableau moderniste à rebours, créant un espace de signes mouvants et tangibles (voire tactiles), fort du travail de la forme, de la couleur et de la toile elle-même. L'usage de la toile de jute, corpulente et sujette à manipulations diverses (comme chez un Alberto Burri par exemple), offre aux premières œuvres de Cherkaoui un champ propice à des gestes d'artisan, voire de paysan : déchirures, coutures et collures dominent le fond de la composition par fragments et couches de toile de jute. L'univers de signes ésotériques - dont la signification se révèlent généralement dans un après-coup à la jouissance du signifiant - vient s'y apposer, bien loin de tout effet calligraphique. Ils semblent alterner en permanence entre transparence et opacité, incorporation et transpiration, mais aussi tout simplement entre écriture et image. A la même époque où s'illustrent les thèses de Clement Greenberg sur la supposée pureté du tableau moderniste, ${ }^{12}$ tout chez Cherkaoui semble faire contre-feux ; au tableau comme surface, il oppose le tableau en quête de corporéité.

Côté marocain, plusieurs auteurs et critiques d'art se mettent alors à l'unisson pour faire valoir une certaine peinture ou " plastique » du signe. Comme Mohamed Sijelmassi, pour citer le plus connu, avec son ouvrage L'art contemporain au Maroc datant de 1989, où il présente Cherkaoui exactement comme Abdellatif Laâbi présentait les enjeux culturels de la revue Souffles:

L'exemple type de l'artiste pourfendant à la fois l'académisme déjà en vigueur et l'orientalisme folklorique, se référant aux formes de l'art populaire, poterie, bijoux, tapis, et surtout tatouages, qu'il regardait enfant intrigué au visage et sur les mains de sa mère ; bref un répertoire de signes qu'il ne reproduit pas mais dont il explore l'ubiquité en les transposant par des moyens plastiques personnels pour leur donner une nouvelle dimension où se reflètent sa méditation, son mysticisme et sa maîtrise du « discours » plastique contemporain. ${ }^{13}$

A presque vingt-cinq ans d'écart, le paradigme est toujours le même que dans le premier numéro de Souffles. Celui d'une rédemption de la tradition à travers le modernisme qui passe par une mise en mouvement, une dynamique (y compris corporelle) des structures ou des motifs ornementaux. C'est dire l'importance de cette revue à son époque et au-delà. Un modernisme rédempteur se nourrissant en somme d'un ensemble d'ornements transfrontières à fortiori éprouvés par leur utilisateur de manière physique, intime, voire mystique ou ésotérique.

12 Greenberg 1961.

13 Sijelmassi 1989: 116. 
Si les signes affichés par les peintures de Cherkaoui semblent se vouloir impénétrables, l'impression est renforcée par son recours à l'alphabet tifinagh utilisé dans la langue amazigh et la culture berbère. Or Cherkaoui n'en demeure pas moins un artiste emblématique d'une modernité cosmopolite liée à Paris, où il étudie aux Beaux-arts, découvre Roger Bissière et Paul Klee qui le marqueront à vie, et finit même par exposer aux côtés d'André Masson et Henri Michaux. Malgré sa connaissance profonde de la calligraphie et de l'écriture sacrée, sur laquelle les commentateurs de Cherkaoui insistent sans faute, il est d'autant plus remarquable que le modernisme rédempteur de ce dernier se soit attaché à des sources préislamiques. Le potentiel graphique et plastique de l'alphabet tifinagh, de ses formes géométriques, anguleuses et " tribales », se distingue de la calligraphie arabe, toute en volutes et en arabesques, semble mieux se prêter au dialogue formel entre Cherkaoui et certains artistes issus de l'Ecole de Paris. Par ailleurs, le choix des sources préislamiques pourrait bien signifier pour lui (au-delà de ses propres origines berbères), une immersion dans un temps lointain, incorruptible à l'épreuve moderniste et à l'occidentalisation, tragique comme un rite initiatique dédié à une langue perdue ; ou réputée disparue depuis l'Antiquité (probablement d'origine phénicienne) mais conservée dans la sphère linguistique touareg. Encore aujourd'hui dans l'inconscient collectif marocain, le tifinagh représente vraisemblablement plus une codification alphabétique, à tout le moins un système de signes graphiques et visuels, avant d'être une "langue " stricto sensu. Et ce alors même que le tifinagh est utilisé dans l'espace public de certaines régions de Kabylie notamment, sans compter les réformes plus récentes contribuant à le réintroduire dans les grandes villes, dans la signalétique urbaine et les opérations administratives. Ce statut hybride a sans aucun doute favorisé sa réappropriation par Cherkaoui et d'autres artistes de la même génération, à l'aune d'une certaine peinture du signe. Une peinture absorbant au passage les enjeux formels et conceptuels de l'avant-garde européenne d'après-guerre (Ecole de Paris, tachisme, abstraction lyrique), dans le jeu archéologique de la trace qui fait signe ou du signe qui fait trace.

De plus, le tifinagh relève d'une tradition vernaculaire et non fixée intégralement dans ses usages, au contraire de la calligraphie. En attestent ses applications relativement anachroniques et divergentes : d'un côté servant chez les Touaregs à écrire des messages circonstanciés ou de brèves conversations inscrites sur les objets intimes comme les bijoux ; d'un autre côté, revendiqué et réintroduit par les militant kabyles, marocains ou algériens, recourant à l'amazigh et au tifinagh pour écrire des chants, des poèmes et des slogans politiques. Récemment encore, l'usage du tifinagh dans l'espace public pouvait être perçu par les autorités marocaines comme une marque 
d'agression ou de subversion. ${ }^{14}$ Une langue "subalterne » en quelque sorte dont les commentateurs de Cherkaoui ont souvent manqué le potentiel politique.

\section{Ornement tatoué}

Les signes de Cherkaoui tendent à s'inscrire sur la toile comme un tatouage marquant une peau, comme si l'imprégnation (ou alternativement l'effacement) du signe se prolongeait au-delà de l'espace pictural où elle a lieu. Un phénomène qui n'a sûrement pas manqué d'inspirer l'écrivain et théoricien de l'image Abdelkébir Khatibi, à commencer par son roman au titre fulgurant $L a$ Mémoire tatouée de 1971. Une odyssée introspective, univers pluridimensionnel où l'auteur évolue au sein d'une infinité d'ornements et autres talismans à déchiffrer ou à expier. Khatibi enlace littéralement son lecteur pour une poignante réappropriation (postcoloniale) du corps, de ses émanations les plus pieuses à ses hallucinations les plus transgressives, érotiques, existentielles :

Me saisit la même fascination devant toute Bédouine tatouée. Quand celle-ci ouvre la main ancestrale, j'épouse ma fixation au mythe. Toute calligraphie éloigne la mort de mon désir, et le tatouage a l'exceptionnel privilège de me préserver. Aucun point de chute dans le chaos, seulement la force d'une impulsion dénouée, un graphe prompt comme un clin d'œil. ${ }^{15}$

Voilà ravivée, en tout point similaire aux « visions » ornementales de Khatibi, la réminiscence maternelle de Cherkaoui évoquée plus haut par Sijelmassi. Certaines des « routes » ésotériques (spirales, hachures, flèches...) qui traversent les toiles de Cherkaoui ou les œuvres sur peau animale de Belkahia (dans deux styles assez différents l'un de l'autre) se retrouvent également sous la plume de Khatibi. Littérature et peinture se sont donc suivies à la trace, évoluant en miroir, de la même attirance pour ces ornements hybrides que représentent les tatouages corporels, les motifs de tapis, les bijoux, le travail du cuivre... Censurés, fétichisés ou dénaturés durant la période coloniale, ils s'offrent à présent une renaissance sous les feux de la conscience postcoloniale ; qu'il s'agisse de formes dérivées de la main de Fatma ou de l'architecture traditionnelle ; des formes dérivées de l'arabesque ou de la spirale, ou encore de l'alphabet tifinagh, la préoccupation fondamentale reste de surmonter, devrait-

14 Becker 2006.

15 Khatibi 2007: 13-14. Voir également, en ce sens, la très belle interprétation des lignes de la main et du paradigme cartographique chez Belkahia, dans Leenhardt, 2001. 
on même dire de "survivre » à la situation décrite ici par le peintre algérien Mohamed Khadda :

\begin{abstract}
Nous avons vu comment, dans les années les plus noires de la colonisation, notre culture et notre art pourchassés, agressés, menacés d'extinction s'étaient, pour survivre, réfugiés, terrés. Mais à force de nuit et d'ombre les formes se sont momifiées, les signes corrodés sont devenus indéchiffrables, les symboles incompréhensibles. ${ }^{16}$
\end{abstract}

Ce sentiment amer de dépossession des signes, que l'on peut également qualifier de processus d'acculturation (généralement propre à la colonisation), devait donc logiquement aboutir dans un mouvement de réappropriation de ces mêmes signes. Poussé notamment par les peintres et les écrivains, ce mouvement que je propose d'appeler modernisme rédempteur est celui dans lequel les signes de la tradition renaissent après la " corrosion ». Mais paradoxalement, le fait qu'ils deviennent à nouveau lisibles ou figurables, ne va pas sans une certaine revendication à l'ésotérisme, comme si le pouvoir des signes une fois ressuscité, il s'agissait dès lors de le préserver de toute nouvelle dénaturation. Une des stratégies qui sera privilégiée, comme nous avons commencé à le voir, est celle de maintenir les signes en mouvement, refusant leur assignation au champ du « décoratif » (qui sous-entend la fixité des signes en jeu). Exactement comme le fait Edmond Amran el-Maleh dans son essai à propos de Cherkaoui, où il explique les buts de l'artiste moderne à travers des préceptes attachés à « l'art islamique », catégorie générique s'il en est, et en réaffirmant l'argument de Oleg Grabar : qu'il n'est pas un art de décoration des surfaces ou de remplissage du vide mais bien un art de transpositions, de combinatoires et de dynamiques spatiales ; engageant en définitive un regardeur actif et non passif. ${ }^{17}$ Pour el-Maleh, par-delà l'usage de l'alphabet tifinagh préislamique, Cherkaoui puise dans l'art islamique ce qui le rend comme Le Livre de sable de J. L. Borges : il ne connaît ni début ni fin et il obsède celui qui le possède jusqu'à le consumer.

Manière de comprendre la position théorique d'el-Maleh non pas seulement comme une interprétation « moderniste » de l'art islamique mais également, et sans doute en premier lieu, comme une tentative de redonner du souffle spirituel et vernaculaire à l'idée de modernisme - par définition profane, cosmopolite et aboutissement d'une occidentalisation culturelle.

16 Khadda 2015.

17 Certaines formules d'el-Maleh confirment ses affinités avec la pensée structuraliste française: " un motif ne signifie que dans sa différence et dans ses rapports avec d'autres " mais aboutissant à des conclusions qui trahissent bel et bien l'enjeu identitaire: « la caractéristique constante de l'art islamique est sa totale liberté, El-Maleh, Maraini, et Khatibi 1976: 18. On lira également le texte de Barthes 1979. 


\section{Ornement didactique}

Pour revenir à l'Ecole de Casablanca, d'un mot, il est temps qu'hommage soit rendu à une de ses figures tutélaires et fondamentales : l'historienne de l'art, anthropologue et romancière italienne Toni Maraini (née en 1941) qui vit au Maroc dans les années 1960-1970. Elle jouera dès la formation du groupe un rôle clé en rédigeant la plupart des textes d'exposition, chroniques et autres manifestes, au sein de l'école d'art de Casablanca, où elle enseigne à partir de 1964 (deux ans après l'arrivée de Belkahia à la direction) ; mais aussi lors des expositions du groupe, comme Belkahia, Chabaâ, Melehi de 1966 dans le hall du théâtre Mohamed V de Rabat. Une exposition retentissante de l'avis de Maraini elle-même, événement fondateur pour ses protagonistes et le public de l'époque ; constitutif d'une nouvelle conscience de la place de l'artiste dans la société, dont on peut lire ici les notes d'intention par Toni Maraini. Le propos se partage entre les dynamiques spatiales de la peinture et ses dynamiques culturelles :

Tandis que l'espace « informel » est sans dimensions, habité par des masses de couleurs flottantes, l'espace ici est actif et dimensionnel : il court se projeter en dehors de la toile.

[...] Si l'on veut faire comme d'autres pays du tiers-monde (valoriser nos racines culturelles) il faut regarder le passé esthétique et le renouveler, or, ce passé arabe, berbère, saharien, est très riche et essentiellement non-figuratif ; cette "disposition à l'abstrait » (géométrique et symbolique) est donc un élément important. Le peintre peut l'intégrer à sa recherche et, conscient de ce passé, se projeter vers le futur pour participer aux investigations modernes à côté des autres pays. C'est dans ce cadre général que se situe la peinture de Farid Belkahia, Mohamed Chabâa, Mohamed Melehi. ${ }^{18}$

Farid Belkahia lui-même devait résumer plus tard ces enjeux d'une maxime désormais célèbre qui renoue avec le texte de Toni Maraini : " La tradition est le futur de l'homme ». Mais l'exposition au théâtre Mohamed V inaugurait également une sorte de brèche dans les modes d'exposition, un lieu pour ainsi dire semi-public semi-privé, dénotant à l'époque le désir de démocratisation de l'art, alors même que les galeries d'art étaient encore inexistantes et que les halles de théâtre ou de cinéma, ainsi que les espaces hôteliers, allaient devenir les seuls lieux d'exposition. Toni Maraini insiste dès lors dans ses témoignages sur le rôle clé de la galerie L'Atelier, première du genre. Fondée par Pauline Chéréméteff de Mazières (plus connue sous le nom de Pauline de Mazières) en 1970 à Rabat - avec la collaboration active de Sylvia Frei-Belhassan à partir de

18 Exposition Belkahia, Chabâa, Melehi dans le hall du théâtre Mohammed V de Rabat. Vernissage le 19 janvier 1966 à 19h30. Archives Toni Maraini. Voir également Maraini 2014. 
1974 - L’Atelier représente un repère, encore trop méconnu, dans l'histoire de l'art moderne et contemporain au Maroc. La galerie, qui sera active jusqu'en 1991, expose les artistes de l'Ecole de Casablanca à plusieurs reprises, toujours aux côtés de Toni Maraini. ${ }^{19}$

Lorsqu'il arrive en tant que jeune directeur de l'Ecole de Casablanca en 1962, Belkahia fait germer l'idée, qu'il poursuivra jusqu'à la fin de sa mission, d'explorer les potentialités des arts traditionnels, des arts populaires et de l'artisanat marocain au bénéfice d'un art « moderne » ou " contemporain » (les appellations diffèrent souvent sur la longue période d'activité de ces artistes). Ce recentrement sur l'identité artistique marocaine comme éthique de la modernité, en insistant toujours sur sa plasticité, sa capacité de transmutation et son lien avec le hors-champ de la peinture, répond tout à fait aux préoccupations de Mohamed Khadda en Algérie, comme vu plus haut, et à ce que Jacques Berque décrit comme « la déperdition culturelle, le trouble de la personne, l'effacement du spécifique ${ }^{20} »$ dans l'expérience coloniale.

Dédiant une grande partie de son énergie à fédérer les initiatives et les savoirs des professeurs et des étudiants, Belkahia soutient et encadre des projets comme la publication Maghreb Art, dirigée par le collectionneur et expert Bert Flint, avec la complicité de Toni Maraini. ${ }^{21}$ Résolument ancrée dans la redécouverte et l'analyse de "l'art populaire au Maroc », Maghreb Art coupe définitivement le cordon avec l'enseignement artistique prodigué sous le Protectorat, considéré comme l'arme « civilisatrice » du pouvoir colonial, un enseignement élitiste et bon pour les étrangers. L'introduction du deuxième volume de cette publication, datant de l'automne 1966 (tout comme l'exposition Belkahia, Chabâa, Melehi au théâtre Mohamed V de Rabat), proclame ainsi :

Il fallait, au lendemain de l'indépendance, reconvertir cet enseignement et rechercher les moyens de l'adapter à nos exigences et à nos réalités. Ainsi avant de faire partie du patrimoine universel, l'art japonais ou l'art mexicain ont d'abord jailli d'un fond de valeurs

19 Entre avril 1971 et décembre 1991, au 6 bis rue d'Annaba à Rabat, la galerie L'Atelier, sans mécénat ni subventions de l'Etat, organise 79 expositions individuelles et 16 expositions collectives, d'artistes d'horizons aussi divers que Mohamed Melehi, Farid Belkahia, Mohamed Chabâa, Mohamed Kacimi, Mohamed Abouelouakar, Rachid Koraïchi, Hossein Zenderoudi, Dia al-Azzawi, Burhan Dogançay, Etel Adnan....

20 Berque 1975: 35.

21 Précisons également le rôle fondamental de Mohamed Melehi en tant que photographe de sites patrimoniaux et de peintures décoratives traditionnelles (notamment dans le Haut-Atlas et l'Anti-Atlas). On lui doit ainsi les illustrations de Maghreb Art qui permettent aux étudiants de se familiariser avec un héritage méconnu (Toni Maraini parle également de visites exploratoires avec les étudiants de l'école). 
collectives. Nous nous trouvons confrontés au Maroc avec cette première phase de regroupement, de revalorisation, temps d'arrêt nécessaire qui doit permettre d'inventorier un langage plastique traditionnel afin de le remettre en évidence et de le replacer dans son contexte dynamique moderne. ${ }^{22}$

Replacer la tradition dans son contexte dynamique moderne pouvait, par exemple, se faire en allant découvrir les peintures ornant les plafonds et piliers de l'architecture de Souss (Anti-Atlas). Bert Flint, fort d'une analyse très documentée, se permet même de les rattacher, par associations visuelles, à l'archéologie mésopotamienne ou à l'Europe du Moyen-Age. Un vocabulaire vernaculaire, composé de surfaces hachurées, de triangles, de losanges et de demi-cercles, qui n'est pas sans nous frapper par sa ressemblance formelle avec l'alphabet tifinagh. A peine quelques années plus tard, ces codes graphiques allaient se retrouver employés de manière systématique et syncrétique dans les œuvres sur peau de Belkahia. Un « système » de signes, largement ésotériques, qui ne cherchent en rien à s'ériger en symboles mais plutôt à sonder une identité composite, suivant justement sa décomposition en traces éparses et parfois très reculées dans le temps. Une identité-palimpseste. Rétrospectivement, le dialogue implicite entre les recherches de Flint et les expérimentations de Belkahia est d'une éloquence et d'une agilité remarquables. Ainsi des nombreuses flèches, spirales et autres continents à la dérive qui peuplent son univers babélien et qui, au contraire d'encourager à la dispersion ornementale, tendent irrémédiablement vers un centre déductible, bien que parfois invisible. Comme si ces flèches et leurs dérivés tentaculaires indiquaient, sans jamais vraiment les fixer, mais tout en laissant deviner un chemin possible de l'un à l'autre, la tradition berbère, la tradition islamique, l'art populaire marocain, les influences sahariennes et africaines. ${ }^{23}$ Sans oppositions foncières, plutôt par cycles et ressacs.

Si les artistes de l'Ecole de Casablanca ont su développer leur travail sur le langage de la peinture dans les conditions historiques liées à la révolution culturelle que représente la conscience postcoloniale, c'est en passant par une certaine dynamique de l'ornement mais encore davantage par une philosophie de l'espace. Le langage babélien des fresques immémoriales " tatouées " par Belkahia, les arabesques «pop » des paysage-haïkus de Melehi ou les labyrinthes géométriques des architectures utopiques peintes par Chabâa... chacun à sa manière, incarne un trait d'union entre l'espace de l'œuvre et l'espace public.

22 Flint et Maraini 1966.

23 Nous reprenons ici les catégories dégagées dans Flint et Maraini 1966. 
L'espace public où le dialogue des signes est le plus fertile se retrouve inscrit dans les interstices du tableau - ou, vice-versa, le tableau s'inscrit dans une architecture imaginaire. "L'Ecole de Casa » incarne un trait d'union entre le patrimoine matériel et le patrimoine immatériel ou encore entre les voyages réels et les voyages imaginaires où ces artistes se sont forgés leur empreinte, leur « ornement » de la modernité.

\section{Rues de Babel}

Dans sa période dite " expressionniste » de voyageur précoce, Farid Belkahia, grandit artistiquement à l'époque où les ondes de choc de la Guerre froide pouvaient rejoindre le continent africain ; exportation intense du conflit idéologique dans les pays du tiers-monde via la diffusion des idées marxistes notamment. Fruit d'un cosmopolitisme grandissant dans lequel les échanges culturels et artistiques entre "périphéries » et " centres " se multiplient, le parcours de Belkahia l'amène aussi bien à la Biennale du Caire, aussi tôt que 1959, dès 1961 à la Biennale de Paris, plus tard à la Biennale de Sao Paulo en 1987 ou celle de La Havane en 1989.

C'est de retour de ses nombreux voyages entre les années 1950 et 1960, suite à ses études à Paris puis à Prague, que Belkahia, accompagné de ses collègues suscités, affute une véritable contre-attaque dans les usages sociaux de l'art ; dans la société marocaine postcoloniale, où la peinture de salon a encore main mise sur tout autre mode d'expression pictural. La génération « Casa » accomplit un de ses grands faits d'armes avec l'exposition - ou plus justement dénommé « Action plastique » - de la place Jemaa el-Fna à Marrakech, le 9 mai 1969. Mohammed Ataallah, Mohammed Chabâa, Mustapha Hafid, Mohammed Hamidi, Mohammed Melehi et Farid Belkahia inaugurent la première manifestation constituée en dehors des salons (ou des ambassades étrangères), littéralement dans la rue. Un marqueur prépondérant dans l'histoire de la peinture moderne au Maroc. L'art prend la parole publiquement, accroché à l'air libre et par l'intermédiaire de la plume du groupe, Toni Maraini :

A Jemaa el-Fna, à Marrakech, se déroulent à longueur de journées différents spectacles populaires. Dans cette atmosphère collective les gens (de la ville, de la campagne, de toutes les couches sociales) se promènent dans un état d'âme particulier. Nous avons accroché nos travaux dans cette place pendant dix jours. Nous avons voulu rejoindre le public populaire là où il se trouve, disponible et décontracté, et nous lui avons proposé cette manifestation vivante : des tableaux exposés à l'air libre, dans une place publique. Des travaux en dehors des cercles fermés des galeries, des salons, dans lesquels ce public n'est d'ailleurs jamais rentré, ne s'est jamais senti concerné par ce genre de manifestation 
en vase clos. Des travaux qui subissent les mêmes variations atmosphériques que les gens, les murs, la place entière. ${ }^{24}$

De la peinture en outil de lien social, instrument dans la lutte des classes à la peinture en écosystème, à la limite éphémère voire éphéméride : sur certaines des photographies immortalisant l'événement, notamment des vues éloignées capturant la place Jemaa el-Fna fourmillante, les peintures accrochées sur les murs extérieurs du bâtiment central se détachent dans le paysage telles des signes cartographiques, des manifestations directes de la migration du motif chère à Abdelkébir Khatibi. On peut également analyser ladite action plastique à la fois comme l'aboutissement d'une subversion méthodique à l'encontre de la peinture de chevalet et comme l'acte de naissance d'une spécificité marocaine dans le modernisme transméditerranéen : cette recherche assidue et partagée entre plusieurs générations d'artistes vers une troisième voie, transcendant les limites de la peinture et de la sculpture, de la surface et de la profondeur mais aussi du mur et du socle.

Quant à l'écosystème de la place Jemaa el-Fna elle-même, s'y rencontrent à l'époque aussi bien les gens des régions du Drâa, du Souss, des descendants de Maures andalous et des gens des tribus Rehâmna venus des plaines voisines. On y entend parler berbère mais on rencontre également des Sahariens, un héritage africain propre à cette place-carrefour que Belkahia se remémore volontiers :

\begin{abstract}
D’abord je suis africain [...] Effectivement, j'ai été profondément marqué par mes voyages en Afrique subsaharienne (le Mali, le Niger, le Sénégal...) dans les années 1970, 1968 je crois. Je n'oublie pas, en tant que Marocain et Africain, toute la culture des caravanes, tout l'échange intense que le Maroc avait avec l'Afrique. Marrakech était, et demeure je suppose, un centre important de ce brassage. La colonisation occidentale et française essentiellement a commencé par couper nos liens avec l'Afrique en coupant les routes caravaniennes, puis en essayant de diriger notre attention vers d'autres régions du monde. ${ }^{25}$
\end{abstract}

Or ce concept de la route déviée ou dérivée, animée par le sentiment d'une origine perdue se cherchant un nouveau futur, Belkahia va le multiplier sous différentes formes dans ses œuvres de tous registres et de tous matériaux. Dans presque chacun de ses grands cycles de travail (les Féminités, les Transes, les Processions, les Cartes, les Mains...) les tableaux-corps (sur peau ou en cuivre principalement) prennent des formes volontairement insaisissables, dignes d'un kamasoutra ou d'un bestiaire borgésien, favorisent des tourbillons de signes et autres constructions babéliennes. On ne sait plus si suivre les routes dessinées

24 Manifeste de l'exposition de la place Jemaa el-Fna, 1969. Archives Toni Maraini.

25 El-Aroussi 2012: 63. 
sur la peau ou bien celles qui se dessinent par le contour polymorphe de l'œuvre. Le motif du labyrinthe ou du continent à la dérive portent les stigmates métaphoriques de ces glissements. De même, tous les fragments de langue ou de codes syncrétiques développées par l'artiste et qui suivent des routes imprévisibles, spiraloïdes, extatiques, comme les énumère Abdelkébir Khatibi au sujet de Belkahia :

[...] Signes, symboles, emblèmes, formes nettes, pictogrammes oubliés, diagrammes sexuels ou astraux. Aide-mémoire qui lui sert d'imagerie, de rêverie sur des traces archétypales, et dont il fait un puzzle ou une série, où le joueur (l'artiste) est en même temps partie du jeu. ${ }^{26}$

Les frontières de la peinture et de la sculpture ne seront que d'autant plus dissoutes, à mesure que se développe l'œuvre de Belkahia, que ce soit à travers les cuivres, les peaux, etc... se détachant toujours autant que possible du tableau au sens moderniste, pour s'approcher d'un modèle directement lié au corps ; dans une conception à fortiori non-anthropomorphique du corps, aux qualités héritées aussi bien de l'histoire naturelle, des gestes calligraphiques ancrés dans une mémoire corporelle (lignes de la main ou ornementations de la main de Fatma, motifs tatoués au henné...), mais aussi de systèmes talismaniques et numérologiques. Chez Belkahia comme chez Cherkaoui la fonction ésotérique de l'ornement tend moins au voilement du sens qu'à sa mise en mouvement, sa démultiplication, sa corporéité.

Le dialogue implicite entre Khatibi (notamment le Khatibi conteur et romancier) et Belkahia ne manque pas d'éloquence ni de ressource, là encore comme avec Cherkaoui dans un hymne à la Mémoire tatouée. Les " routes » spiraloïdes ou en zigzags de Belkahia distillent en réalité un sentiment spatial bien proche de celui qui animait la « prise » de la place Jemaa el-Fna. A savoir favoriser le lien et la mixité sociale, la transpiration des signes et la porosité des identités dans l'espace public ; jouer des strates de sens et d'expressions corporelles nées de l'agitation sur cette place ou dans les ruelles tortueuses de la médina ; contrecarrer en somme l'imagination urbaine coloniale qui, elle, consiste à « juxtaposer, compartimenter, militariser, découper la ville en zones ethniques, ensabler la culture du peuple dominé-27 ${ }^{27}$.

Le monde philosophique autonome recomposé par Belkahia, au travers de son langage ésotérique et babélien, fait de lui l'artiste transfrontières qui navigue entre ses affinités électives avec sa capacité à les transcender en permanence. En un certain sens, il rencontre la condition d'un spectateur postmoderne pour qui les signes ne sont plus le simple réceptacle d'un dévoilement

26 Khatibi 2001: 112. 
épiphanique mais la nouvelle monnaie d'échanges dans les enjeux de traductibilité que nous vivons, d'un espace l'autre. De moins en moins confortés dans une langue et de plus en plus confrontés à une multiplicité d'ensembles signifiants, nous traitons de "sources » et de " codes " informatiques. Cette mémoire virtuelle pourrait bien engloutir notre mémoire des signes, ainsi que le vieux concept de bibliothèque ou d'encyclopédie, sous le spectre des archives numériques. A moins que celles-ci ne recèlent d'autres ornements encore indéchiffrables à cette heure.

\section{Bibliographie}

El-Aroussi, Moulim (2012) : « Entretien avec Farid Belkahia ». In : Identité et modernité dans la peinture marocaine. Casablanca : Loft Art Gallery.

Barthes, Roland (1979) : «Ce que je dois à Khatibi » (in Khatibi, Abdelkébir (1979) : La Mémoire tatouée, Paris : Union générale d'éditions), in Barthes, Roland (2002) : Fuvres complètes, Paris : Éditions du Seuil, vol. 5, 667.

Berque, Jacques (1975) : Langages arabes du Présent. Paris : Gallimard.

Becker, Cynthia (2006) : Amazigh Arts in Morocco. Women Shaping Berber Identity. Austin, TX : Texas University Press.

Buci-Glucksmann, Christine (2008) : Philosophie de l'ornement. D'Orient en Occident. Paris : Galilée.

Flint, Bert / Maraini, Toni (1966) : «Introduction ». Maghreb Art, n², automne 1966, Ecole des Beaux-arts de Casablanca.

Focillon, Henri (2004) : Vie des formes. Paris : PUF (1943).

Golsenne, Thomas (2010) : "L'ornement a horreur de l'horreur du vide ». Zamân (Textes, images et documents) $3: 112-113$.

Golsenne, Thomas (2012) : « L'ornement aujourd'hui ». In : Images Re-vues [en ligne], n¹0, URL : http ://imagesrevues.revues.org/2416.

Greenberg, Clement (1961), Art and Culture. Boston, MA : Beacon Press.

Khadda, Mohamed (2015) : Eléments pour un art nouveau. Suivi de Feuillets épars liés et Inédits. Alger : Barzakh.

Khatibi, Abdelkébir (1987) : Figures de l'étranger dans la littérature française. Paris : Denoël.

Khatibi, Abdelkébir (2001) : L'art contemporain arabe. Paris : Al Manar-Institut du monde arabe. Khatibi, Abdelkébir (2007) : La Mémoire tatouée. El-Jadida : Okad (1971).

Leenhardt, Jacques (2001) : « Le Maître du schème ». In : Farid Belkahia, livret d'exposition, 3 mars-30 mai 200. Marrakech : Musée de Marrakech.

El-Maleh, Edmond Amran / Maraini, Toni / Khatibi, Abdelkébir (1976) : La Peinture de Ahmed Cherkaoui. Casablanca : Shoof.

Naef, Silvia (1996) : A la recherche d'une modernité arabe. L'évolution des arts plastiques en Egypte, au Liban et en Irak. Genève : Slatkine.

27 Khatibi 2007: 46 
Riegl, Aloïs (2002) : Questions de style. Fondements d'une histoire de l'ornementation. Paris : Hazan.

Sefrioui, Kenza (2013) : La revue Souffles. 1966-1973. Espoirs de révolution culturelle au Maroc. Casablanca : Sirocco.

Shabout, Nada (2007) : Modern Arab Art. Formation of Arab Aesthetics. University Press of Florida.

Sijelmassi, Mohamed (1989) : L'art contemporain au Maroc. Courbevoie : ACR. 